Check for updates

Cite this: RSC Adv., 2017, 7, 28931

Received 2nd March 2017

Accepted 26th May 2017

DOI: $10.1039 / c 7 r a 02562 a$

rsc.li/rsc-advances

\section{Comparative suppression of NLRP3 inflammasome activation with LPS-induced inflammation by blueberry extracts (Vaccinium spp.)}

\begin{abstract}
Huailing Wang, ${ }^{a}$ Xinbo Guo, (DD *ab Jie Liu, ${ }^{c}$ Tong Li, ${ }^{b}$ Xiong Fu ${ }^{a}$ and Rui Hai Liu*b
The aim of this study was to evaluate the anti-inflammation effects of blueberry extracts through NLRP3 inflammasome. The anti-inflammation activities of blueberry extracts were detected using lipopolysaccharide (LPS) activated the mononuclear macrophage (RAW264.7), and then detected the gene expression levels of NLRP3, Caspase-1, apoptosis-associated specked-like protein (ASC) and inflammatory factor including $I L-1 \beta, T N F-\alpha, I L-6$, and iNOS. Blueberry extracts could significantly inhibit the gene expression of $I L-1 \beta, T N F-\alpha, I L-6$, and $i N O S$, but the inhibitions were showed differently among the varieties of Blomidom (BD), North Country (NC) and JK-M7 (JK). The protein expressions of NLRP3 and Caspase-1 in the blueberry treated groups were significantly lower than the LPS treated group. Quercetin significantly inhibited LPS-induced ROS and inhibited the mRNA expression and protein levels of NLRP3 and Caspase-1 in RAW264.7 cells.
\end{abstract}

\section{Introduction}

In recent years there have been many epidemiological and clinical studies ${ }^{\mathbf{1}}$ which have indicated that chronic inflammation is a significant risk factor in various human diseases, in particular cardiovascular diseases, diabetics and cancer. ${ }^{2,3}$ In terms of human immune-response, the innate immunity is the first defence against pathogens (such as bacteria, fungi, and viruses, etc.) discriminating "self" from "non-self". The innate immune system utilizes an array of germline-encoded patternrecognition receptors (PRRs) to detect invariant microbial attacks. These recognize situations of host danger promoting activation of the immune system and tissue repair in response to infection or injury. In such a situation the host secret several pro-inflammatory cytokines such as TNF- $\alpha$, IL-1, IL-6, IL-8 and IL-12, and the anti-inflammatory cytokines such as IL-4, IL-10, IL-11 as well as transforming growth factor- $\beta$ (TGF- $\beta$ ). The cytokines keep the balances in the body to play central roles in mediating and modulating inflammation.

This activation is critical for pathogen clearance and the induction of an adaptive immune response. ${ }^{2}$ The central roles of the NLR family (including NLRP1, NLRP3, and NLRPC4) in the immune system has become increasingly noticed in recent years. ${ }^{4,5}$ One of the best characterized inflammasome is NLRP3,

${ }^{a}$ School of Food Science and Engineering, South China University of Technology, Guangzhou 510641, China. E-mail: xbg720@gmail.com; Tel: +86-20-87113848

${ }^{b}$ Department of Food Science, Stocking Hall, Cornell University, Ithaca, New York 14853, USA. E-mail: rl23@cornell.edu; Tel: +1-607-255-6235

${ }^{c}$ Institute of Traditional Chinese Medicine and Natural Products, College of Pharmacy, Jinan University, Guangzhou 510632, China also known as CIAS1, CRYOPYRIN, NALP3 or PYPAF. This substance is encoded by the NLRP3 (NOD-like receptor family, pyrin domain) gene in humans. NLRP3-mediated inflammasome activation occurs in response to diverse molecular entities of bacteria, ${ }^{6}$ viruses, ${ }^{7,8}$ fungi, ${ }^{9}$ dying cells, ${ }^{6}$ crystal particles, ${ }^{10-13}$ and DNA. ${ }^{14}$ Normal activation of the NLRP3 inflammasome contributes to host defence. However, excessive activation of the NLRP3 signalling leads to the pathogenesis of a spectrum of auto-inflammatory diseases. ${ }^{15,16}$

The NLRP3 inflammasome is a multi-protein complex that could trigger the maturation of the pro-inflammatory cytokines IL-1 $\beta$ and IL-18.,17 Upon activation, NLRs recruit adaptor ASC and effector Caspase-1 to form large cytoplasmic complexes, defined as the inflammasome. ${ }^{18}$ The encoded protein may play a role in the regulation of inflammation and apoptosis. Consistent with an essential role for NLRP3 inflammasome in antifungal immunity, Gross et al. ${ }^{9}$ showed that NLRP3-deficient mice were hyper-susceptible to $C$. albicans infection. Activation of the NLRP3 inflammasome in response to bacteria, viruses or to RNA/DNA was dependent upon liposomal maturation and reactive oxygen species production in human cells. It has been suggested that the NLRP3 inflammasome senses obesityassociated danger signals and contributes to obesity-induced inflammation and insulin resistance accumulation of inflammation markers in these tissues. ${ }^{19,20}$ Therefore, NLRP3 inflammasome signalling is regulated tightly to maintain immune balance. ${ }^{6,21}$

Reports $^{22}$ had been suggested that the activated of the NLRP3 through 3 signals as the following: the ATP or nigericin could induce mitochondrial dysfunction, which contributes to NLRP3 inflammasome activation through release of mtDAMP, such as 
mtDNA and mtROS, or by inducing a borning mitochondrial movement. The second signal was that the activation of NLRP3 inflammasome which was related with the TLR receptors through the nuclear factor-kappa B (NF- $\kappa \mathrm{B})$ signal transduction pathway. The last signal was that the mitochondrial fusion protein 2 (Mfn2), antiviral immune signalling pathway junction protein (MAVS) and the mitochondrial membrane cardiolipin together recruit the NLRP3 and the NLRP3 protein, combine the ASC and active the procaspase- 1 protein to assemble the NLRP3 inflammasome, which lead to the pro-inflammatory process through the production of IL-1 $\beta$ or IL-18. Many reports ${ }^{23,24}$ indicate that the microbial products, such as nigericin, maitotoxin, hemolysin, or bacterial RNA, induce the robust secretion of IL-1 $\beta$ from LPS-primed bone marrow-derived macrophages (BMDNs) in an NLRP3-dependent manner. To our knowledge, this was the first reports that the blueberry extracts and quercetin exhibited anti-inflammation activity through inhibiting the produce of the NLRP3 inflammasome.

Blueberries are cultivated widely throughout the world ranking behind strawberries in terms of total production ${ }^{25}$ and belong to the family of Ericaceae Vaccinium. Blueberries are popular in terms of taste and are rich in anthocyanin including (cyanidin chloride, petunidin chloride, peonidin chloride, delphinidin, Malvin), phenolics and flavonoids. ${ }^{26}$ These compounds have been studied in relation to the biological activity of blueberry extracts in mediating antitumor activity, ${ }^{27}$ antioxidant activity, ${ }^{28,29}$ and innate and or adaptive immune system. ${ }^{30}$ The increased consumption of fruits has been correlated with health benefits and linked with decreased incidence of several diseases, including metabolic syndrome, ${ }^{31}$ and diabetes. ${ }^{2,32}$

Blueberries have been shown to block the nuclear translocation of p65 mediated by NF- $\kappa$ B pathway. ${ }^{33}$ This is associated with inhibition of TNF- $\alpha$-induced inflammatory responses, ${ }^{34}$ and potential stimulation of anti-inflammatory activity against interleukin-8 (IL-8) for inhibited of matrix metalloproteinase-1 (MMP-1) expression. ${ }^{35}$ The anthocyanins of blueberries have been shown to reduce the activation of NF- $\kappa \mathrm{B}$, induced by IL-1 $\beta$ in intestinal epithelial Caco-2 cells. ${ }^{36}$ These results support that blueberry bioactive compounds have potential bioactivities to act as dietary anti-inflammatory agents. Many researchers have reported that quercetin is a strong antioxidant in fruits. ${ }^{37,38}$ Though blueberry has been extensively consumed, the detailed molecular mechanism of actions is only being explored in recent years.

Previous studies suggested that NLRP3 inflammasome activity is negatively regulated by autophagy and positively regulated by reactive oxygen species (ROS) derived from an uncharacterized organelle. As the molecular mechanisms by which NLRP3 agonists induce mitochondrial ROS production and mitochondrial ROS activate the NLRP3 inflammasome are unknown, the relationship of the ROS and the NLRP3 need to be investigated. In this manuscript, the RAW264.7 cells were stimulated by LPS to produce NLRP3 inflammasome, and the expression of the mRNA and protein level in the stimulated cells was determined to evaluate the anti-inflammation activities of different blueberry varieties.

\section{Results}

\section{Cytotoxicity assay of blueberry extracts}

The cytotoxic effects of blueberry were tested on RAW264.7 cells using the method reported previously. ${ }^{39}$ The cytotoxic effects of quercetin, and blueberry extracts were tested on RAW264.7 cells line as shown in Fig. 1. The $\mathrm{CC}_{50}$ value of the standard was quercetin $\left(241.0 \pm 7.2 \mu \mathrm{mol} \mathrm{L}{ }^{-1}\right)$. The $\mathrm{CC}_{50}$ value of blueberry extracts ranged from $64.32 \pm 3.44$ to $162.5 \pm 4.7 \mathrm{mg} \mathrm{mL}^{-1}$. The lowest $\mathrm{CC}_{50}$ values were found in varieties of $\mathrm{BD}(64.04 \pm$ $\left.5.91 \mathrm{mg} \mathrm{mL}^{-1}\right)$, whilst the variety of $\mathrm{NC}$ was $90.33 \pm 5.83 \mathrm{mg}$ $\mathrm{mL}^{-1}$, and the varieties of JK had the highest values: $162.0 \pm$ $9.5 \mathrm{mg} \mathrm{mL}{ }^{-1}$. Previous reports ${ }^{40}$ have been suggested that the blueberry extracts $\left(400 \mu \mathrm{g} \mathrm{mL}{ }^{-1}\right)$ had highly anti-inflammation effects in RAW264.7 macrophages.

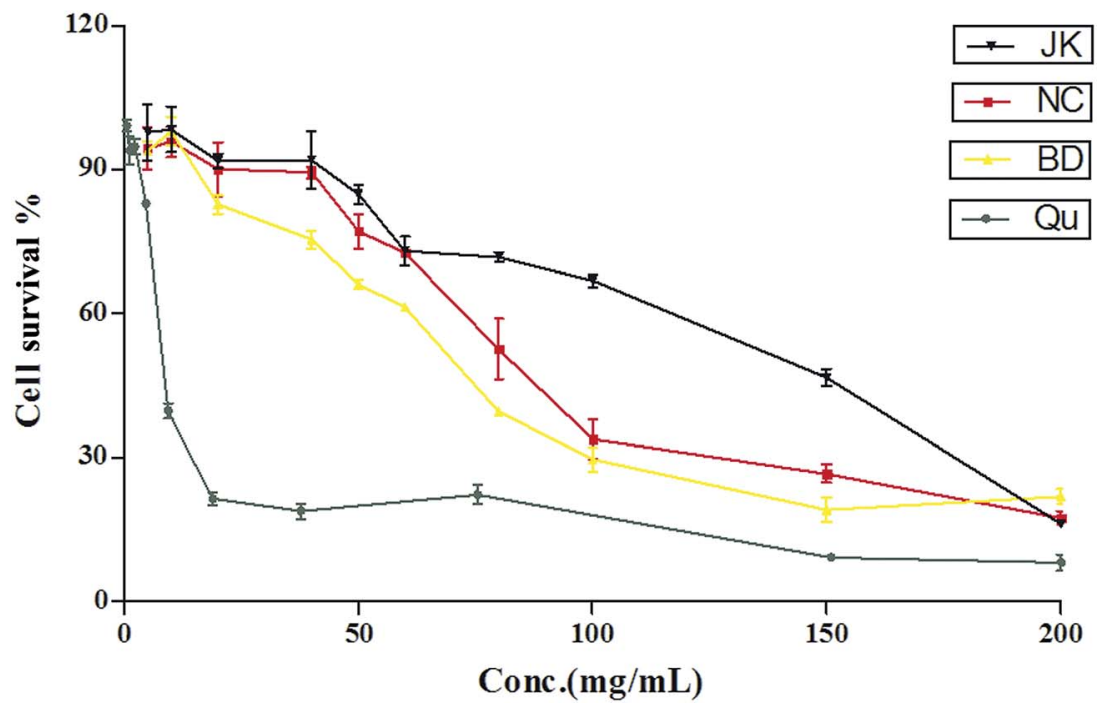

Fig. 1 The cytotoxicity of the blueberry against mononuclear macrophage Raw264.7 cells (mean $\pm S D, n=3$ ). 


\section{ROS detection of blueberry extracts}

To investigate the oxidative damage effect of ROS, the RAW264.7 cells were stimulated with LPS. The blueberry extracts and quercetin dramatically inhibited cellular ROS expression (Fig. 2). The LPS-treated group was regarded as the negative group, and the relative expression of ROS expression was regarded as 1 . The group without blueberry and no LPS regarded as control group, and the relative expression of the ROS was $0.240 \pm 0.097$. Quercetin was used as positive control. The relative expression of the ROS was ranged from $0.407 \pm$ 0.007 to $0.681 \pm 0.023$ in a dose-dependent manner. The varieties of BD showed the lowest relative expression between 0.392 \pm 0.031 and $0.743 \pm 0.021$ with a dose-dependent manner, whilst the JK variety emerged the highest relative expression between $0.911 \pm 0.310$ and $0.982 \pm 0.130$. The BD variety was $0.504 \pm 0.082$ to $0.993 \pm 0.082$.

Based on the experimental results, the $\mathrm{EC}_{50}$ value of the relative expression of ROS was calculated. The value of the NC was $454 \pm 12 \mu \mathrm{g} \mathrm{mL}{ }^{-1}$, and the BD was $483 \pm 19 \mu \mathrm{g} \mathrm{mL}^{-1}$. The $\mathrm{EC}_{50}$ values of JK variety cannot be calculated. The phytochemical compositions in blueberry have been reported ${ }^{\mathbf{4 2}}$ that the varieties of the $\mathrm{NC}, \mathrm{BD}$ and JK contained abundant of phenolics, flavonoids and several anthocyanin. Correlations among cyanidin, delphinidin, malvidin, petunidin, peonidin, $p$ coumaric, caffeic acid, ferulic acid, salicylic acid, quercetin, catechin, resveratrol, relative ROS expression in low concentration (ROS-L), relative ROS expression in middle concentration (ROS-M), relative ROS expression in high concentration (ROS-H) of blueberry were analysed. ROS-H values were highly positive correlation with ferulic acid (0.938), and negative correlation to catechin $(-1.000)$ and petunidin value $(-0.715)$.
ROS-M values were highly correlation with ferulic acid (0.970), and negative correlation to catechin $(-0.995)$ and $p$-coumaric value $(-0.949)$. ROS-L were highly correlated to ferulic acid (0.726), resveratrol (0.904), caffeic acid (0.666) and quercetin $(0.654)$ value, while low correlation with malvidin $(-0.996)$. The results indicated that phenolics played the main roles for in ROS formation. ROS was considered as an oxidization molecule which played an important role in the oxidative process. ${ }^{41}$ In addition to its oxidization effects, ROS has been known as an immune-modulatory molecule, although the mechanisms underlying this immune-modulation are not well understood. In this manuscript, the inflammatory cytokine were detected.

\section{Relative gene expression of inflammatory markers}

LPS is a TLR4 specific agonist that has been used to be a strong inducer of inflammatory responses in macrophages. To investigate whether the production of the cytokine and NLRP3 inflammasome including ASC, NLRP3, IL-1 $\beta$ were influenced by the blueberry extracts, RAW264.7 cells were treated with the certain concentrations of the blueberry extracts and quercetin in the present of LPS. In this experiment, we determined the effect of quercetin and blueberry extracts on the relative

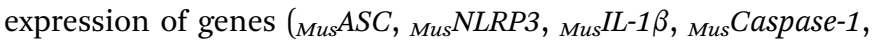
$\left.{ }_{M u s} I L-6,{ }_{M u s} T N F-\alpha,{ }_{M u s} i N O S\right)$, and the results were presented in the Table 2.

The values of the LPS-induced treatment groups were expressed as 1 . The relative expressions of ${ }_{M u s} N L R P 3$ were sharply decreased with the increasing concentrations of the blueberry extracts and the quercetin. In the quercetin treatment groups, the relative expressions of ${ }_{M u s} N L R P 3$ were between 0.515 \pm 0.016 and $0.144 \pm 0.014$, the relative expressions of

\section{ROS}

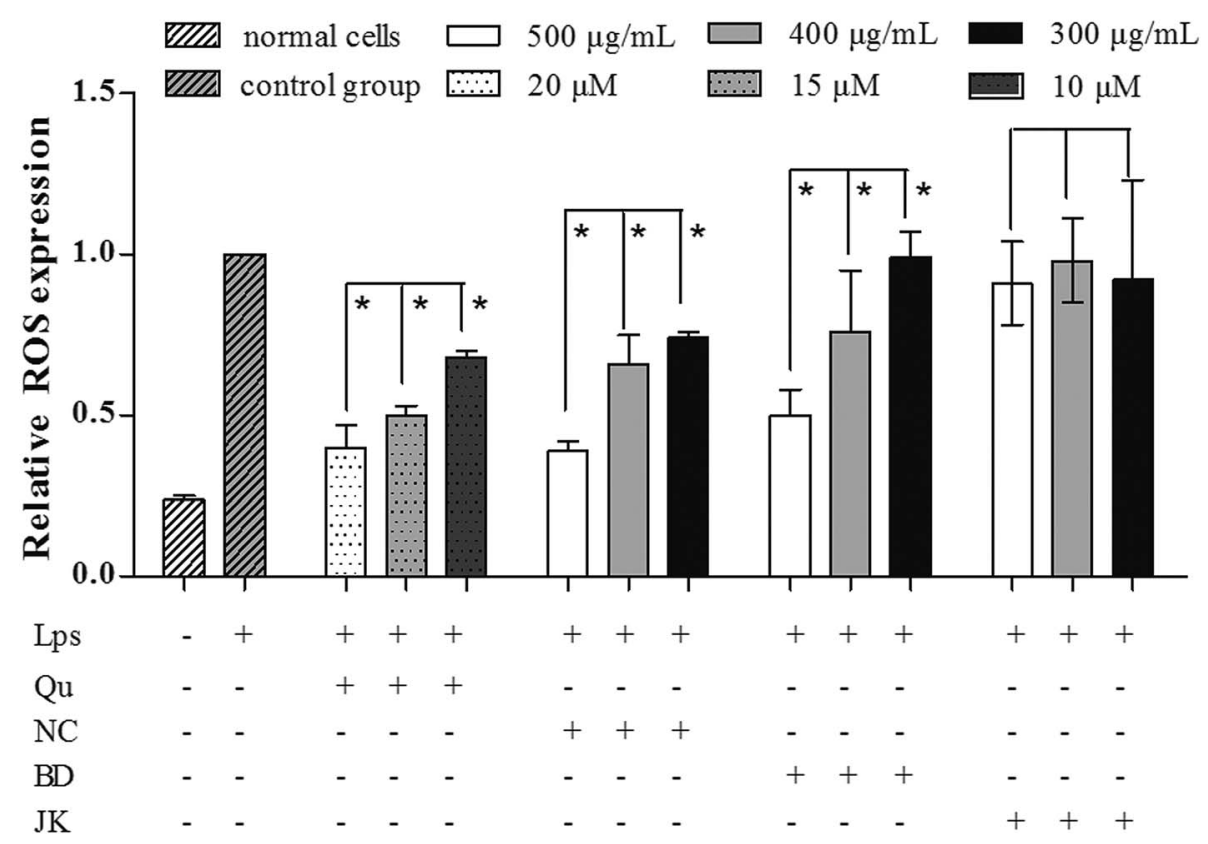

Fig. 2 The relative expression of ROS in mononuclear macrophage Raw264.7 cells (mean $\pm \mathrm{SD}, n=3$ ). 
Table 1 The primer sequences for RT-qPCR

\begin{tabular}{|c|c|c|c|}
\hline Gene & Gene ID & Direction & Primer sequences $\left(5^{\prime}-3^{\prime}\right)$ \\
\hline \multirow[t]{2}{*}{${ }_{M u s} G A P D H$} & \multirow[t]{2}{*}{14433} & Forward & GTCATTGAGAGCAATGCCAG \\
\hline & & Reverse & GTGTTCCTACCCCCAATGTG \\
\hline \multirow[t]{2}{*}{ MusNLRP3 } & \multirow[t]{2}{*}{216799} & Forward & GTGGAGATCCTAGGTTTCTCTG \\
\hline & & Reverse & CAGGATCTCATTCTCTTGGATC \\
\hline \multirow[t]{2}{*}{ Mus Caspase-1 } & \multirow[t]{2}{*}{12362} & Forward & GAGCTGATGTTGACCTCAGAG \\
\hline & & Reverse & CTGTCAGAAGTCTTGTGCTCTG \\
\hline \multirow[t]{2}{*}{${ }_{M u s} I L-1 \beta$} & \multirow[t]{2}{*}{16176} & Forward & GAGCCTGTGTTTCCTCCTTG \\
\hline & & Reverse & TCCAAGAAACCATCTGGCTAGG \\
\hline \multirow[t]{2}{*}{${ }_{M u s} A S C$} & \multirow[t]{2}{*}{66824} & Forward & CTCTGTATGGCAATGTGCTGAC \\
\hline & & Reverse & GAACAAGTTCTTGCAGGTCAG \\
\hline \multirow[t]{2}{*}{ Mus $T N F-\alpha$} & \multirow[t]{2}{*}{21926} & Forward & GGGAGCAAAGGTTCAGTGAT \\
\hline & & Reverse & CCTGGCCTCTCTACCTTGTT \\
\hline \multirow[t]{2}{*}{ Mus $I L-6$} & \multirow[t]{2}{*}{16193} & Forward & CTGACAATATGAATGTTGGG \\
\hline & & Reverse & TCCAAGAAACCATCTGGCTAGG \\
\hline \multirow[t]{2}{*}{ MusiNOS } & \multirow[t]{2}{*}{18126} & Forward & AAGCAGCTGGCCAATGAG \\
\hline & & Reverse & CCCCATAGGAAAAGACTGC \\
\hline
\end{tabular}

${ }_{M u s}$ Caspase- 1 were between $0.521 \pm 0.018$ and $0.358 \pm 0.079$, and the relative expressions of ${ }_{M u s} A S C$ were $0.273 \pm 0.014$ to $0.087 \pm 0.033$. The relative expressions of the other Inflammatory factors were as following: ${ }_{M u s} I L-1 \beta(0.471 \pm 0.081$ to $0.214 \pm$ $0.046),{ }_{M u s} T N F-\alpha(0.462 \pm 0.017$ to $0.321 \pm 0.068),{ }_{M u s} I L-6(0.731$ \pm 0.046 to $0.348 \pm 0.018),{ }_{M u s} i N O S(0.664 \pm 0.035$ to $0.751 \pm$ $0.003)$. The NC variety highly inhibited the expression of the ${ }_{M u s} N L R P 3,{ }_{\text {Mus }}$ Caspase-1, ${ }_{\text {Mus }} I L-1 \beta,{ }_{\text {Mus }} A S C$, ${ }_{\text {Mus }} T N F-\alpha,{ }_{\text {Mus }} I L-6$, and MusiNOS. Meanwhile the varieties of the blueberry extracts including $\mathrm{BD}, \mathrm{NC}$, and $\mathrm{JK}$ had high inhibitory effects to the inflammatory factors. In the NC variety, the relative expressions of ${ }_{M u s} N L R P 3$ were between $0.276 \pm 0.018$ and $0.077 \pm 0.096$, the relative expressions of ${ }_{M u s}$ Caspase- 1 were between $0.240 \pm 0.058$ and $0.210 \pm 0.004$, and the relative expression of ${ }_{M u s} A S C$ was $0.004 \pm 0.001$. The relative expressions of the other Inflammatory factors were as following: ${ }_{M u s} I L-1 \beta(0.028 \pm 0.001$ to $0.002 \pm$ $0.0001),{ }_{M u s} T N F-\alpha(0.682 \pm 0.026$ to $0.287 \pm 0.005),{ }_{M u s} I L-6(0.178$ \pm 0.008 to $0.012 \pm 0.008)$, and ${ }_{M u s} i N O S(0.068 \pm 0.002$ to $0.011 \pm$ $0.001)$. The relative expression $N L R P 3$ mRNA in BD was between $0.408 \pm 0.067$ and $0.196 \pm 0.039$, the relative expressions of Mus Caspase- 1 were between $0.768 \pm 0.014$ and $0.605 \pm 0.035$, and the relative expressions of ${ }_{M u s} A S C$ were $0.272 \pm 0.021$ to $0.158 \pm 0.019$. But the relative expressions of ${ }_{M u s} i N O S$ had no inhibitory effects and the values were higher than the LPStreated group. The relative of expressions of the other inflammatory factors were as following: ${ }_{M u s} I L-1 \beta(0.595 \pm 0.081$ to $0.248 \pm 0.046),{ }_{M u s} T N F-\alpha(0.672 \pm 0.021$ to $0.445 \pm 0.033),{ }_{M u s} I L-$ $6(0.774 \pm 0.013$ to $0.664 \pm 0.035)$. In the JK variety, the relative expressions of ${ }_{M u s} N L R P 3$ were between $0.111 \pm 0.009$ and 0.026 \pm 0.001 , the relative expressions of ${ }_{\text {Mus }}$ Caspase- 1 were between $0.328 \pm 0.032$ and $0.119 \pm 0.004$, and the relative expressions of ${ }_{M u s} A S C$ was only $0.001 \pm 0.0001$. The relative expressions of the other inflammatory factors were as following: ${ }_{M u s} I L-1 \beta(0.234 \pm$ 0.013 to $0.054 \pm 0.029),{ }_{M u s} T N F-\alpha(0.940 \pm 0.059$ to $0.721 \pm$ $0.030),{ }_{M u s} I L-6(0.537 \pm 0.047$ to $0.363 \pm 0.015),{ }_{\text {Mus }} i N O S(0.584 \pm$ 0.021 to $0.364 \pm 0.021)$. In summary, the blueberry extracts and quercetin significantly inhibited the gene expressions of the Mus NLRP3, which was important to the NLRP3 inflammasome, and other inflammatory factors.

Previous reports ${ }^{40}$ suggested that the phenolics from blueberry had significant anti-inflammatory activities which could suppress the gene expressions of ${ }_{M u s} I L-1 \beta$, ${ }_{\text {Mus }} I L-6$, and Mus $I L-12$ p35 in LPS-induced RAW264.7 macrophages. The relative gene expression of ${ }_{M u s} N L R P 3$ was concentration-dependent, and the correlation values were: NC (0.998), BD (0.987), and JK (0.963). The highest inhibitory effects were observed in the

Table 2 Relative gene expression of inflammatory cell model by LPS-induced (mean $\pm \mathrm{SD}, n=3$ ). "ND" means "not detected"

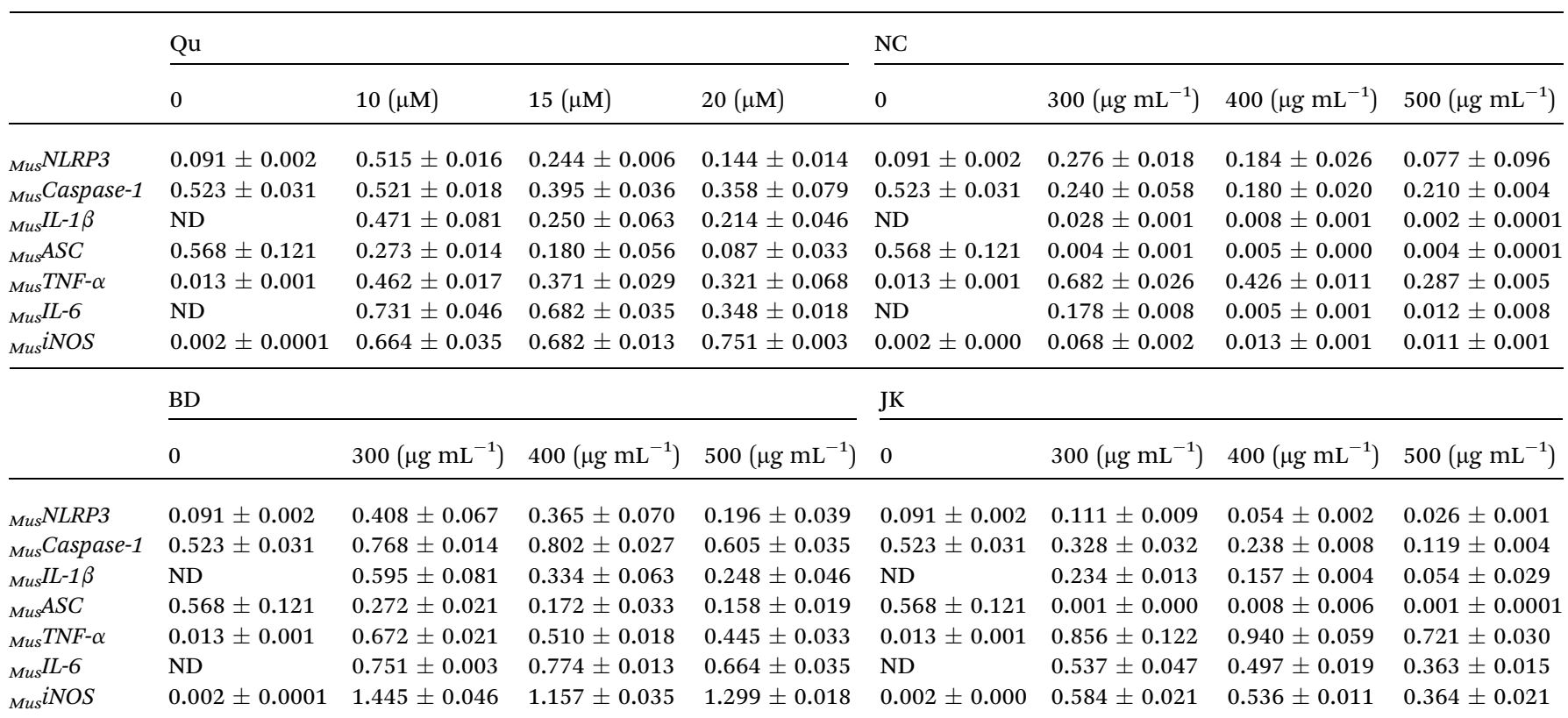


${ }_{\text {Mus }} I L-1 \beta$, and the correlation values were: NC (0.912), BD (0.922), JK (0.993). The correlation values of ${ }_{M u s} T N F-\alpha$ were: NC (0.972), BD (0.943), and JK (0.373). The correlation values of ${ }_{M u s} I L-6$ expression were: NC (0.718), BD (0.562), and JK (0.911). The correlation values of ${ }_{M u s} i N O S$ were as following: NC (0.776), BD (0.257), and JK (0.904). The correlation values of ${ }_{\text {Mus Caspase- } 1}$ were: NC (0.250), BD (0.599), and JK (0.994). The relative expression of ${ }_{M u s} A S C$ did not exhibited concentrationdependent, and the correlation values were: NC (0.001), BD (0.841), JK (0.001).

According to the previously reports ${ }^{42}$ the correlations between phytochemical compositions including cyanidin, delphinidin, malvidin, petunidin, peonidin, $p$-coumaric, caffeic acid, ferulic acid, salicylic acid, quercetin, catechin, and resveratrol, and relative cell factor expressions in different concentrations were analyzed. ${ }_{M u s} N L R P 3$ was highly correlated with salicylic acid (0.822), quercetin (0.765), and caffeic acid (0.760). Mus Caspase-1 was highly correlated with salicylic acid (0.838), quercetin (0.951), caffeic acid (0.953) and resveratrol (0.915). ${ }_{M u s} I L-1 \beta$ was highly correlated with resveratrol (0.854). ${ }_{M u s} A S C$ was highly correlated with salicylic acid (0.850), quercetin (0.954), caffeic acid (0.951) and resveratrol (0.902). ${ }_{\text {Mus }} I L-6$ was highly correlated with resveratrol (0.985). MusiNOS was highly correlated with quercetin (0.886), caffeic acid (0.892) and resveratrol (0.985). Mus TNF- $\alpha$ was correlated with phytochemical compositions, and the best correlation value was 0.749 . The results indicated that phenolic acids (caffeic acid and salicylic acid) and flavonoids (quercetin) and resveratrol from blueberry played the main roles in cell factor expressions.

Phenolic compounds, for example anthocyanin, have been suggested to have an anti-inflammatory effect against LPSinduced changes in immune cells. Black rice extract ${ }^{39}$ could suppress of inflammatory responses via down-regulation of NF$\kappa \mathrm{B}$ and AP-1 signalling pathways. Red bean extracts ${ }^{\mathbf{4 3}}$ were reported to inhibit LPS-induced inflammation and $\mathrm{H}_{2} \mathrm{O}_{2}$-induced oxidative stress in RAW264.7 cells. Johnson et al. ${ }^{37}$ reported that the anthocyanins and proanthocyanidins from blueberryblackberry fermented beverages inhibit markers of inflammation in macrophage. Garcia-Diaz et $a l^{\mathbf{4 0}^{\mathbf{0}}}$ reported that the anthocyanins of fermented berry beverages could inhibit inflammation related adiposity response in vitro. Beneficial effects of berries in the diet have also been attributed to their high phenolic contents. ${ }^{42}$ Research has shown that blueberries could reduce pro-inflammatory cytokine TNF- $\alpha$, and IL-6 production in mouse macrophages by inhibiting NF- $\kappa \mathrm{B}$ activation and the MAPK pathway. ${ }^{\mathbf{4 4}}$

In summary, blueberry extracts and quercetin inhibited expressions of ${ }_{M u s} A S C,{ }_{M u s}$ Caspase-1, and ${ }_{M u s} N L R P 3$, which were the components of the NLRP3 inflammasome and the proinflammatory cytokines including TNF-a, IL-6 and iNOS.

\section{Western blot analysis}

To explore whether the blueberry extracts and the quercetin had an effect on LPS-induced activation of NLRP3 inflammasome, RAW264.7 cells were treated with blueberry extracts and quercetin in the presence or absence of LPS followed by Western blot analysis. Studying the activation of NLRP3 inflammasome, the expression of the Caspase- 1 and the NLRP3 protein and the values of protein relative expression were presented in Fig. 3.

The values of LPS-induced treatment groups were expressed as 1. The NLRP3 expressions of protein levels were sharply decreased with the increasing concentrations of the blueberry extracts and the quercetin. In the $\mathrm{NC}$ variety, the relative protein expressions of NLRP3 were between $0.637 \pm 0.001$ and $0.288 \pm 0.001$, the relative Caspase- 1 protein expressions were between $0.321 \pm 0.001$ and $0.158 \pm 0.001$. The BD variety inhibited the expression of the NLRP3 (0.806 \pm 0.006 to 0.898 $\pm 0.014)$ and Caspase- $1(0.832 \pm 0.011$ to $0.809 \pm 0.008)$. Meanwhile the JK variety had low inhibition effects to the NLRP3 and Caspase-1. In the JK variety, the relative protein expressions of NLRP 3 were between $0.715 \pm 0.003$ and $0.479 \pm$ 0.001 , and the relative protein Caspase-1 expressions were between $1.024 \pm 0.006$ and $0.788 \pm 0.006$. In summary, the blueberry extracts and quercetin could significantly inhibit the expression of the protein NLRP3 and Caspase-1 which were composed of the NLRP3 inflammasome, and other inflammatory factor.

The relative protein expression of NLRP3 was concentrationdependent with the highest inhibitory effects, and the correlation values were: NC (0.999), BD (0.999), and JK (0.970). The higher inhibitory effects were observed in the protein Caspase1 with correlation values: NC (0.964), BD (0.987), and JK (0.930). The correlations between phytochemical composition and relative protein expression of the NLRP3 and Caspase- 1 were analyzed. The relative expression of the NLRP3 values was highly correlated with resveratrol (0.843), quercetin (0.754), and caffeic acid (0.762). The relative protein expression of Caspase-1 values were highly correlated with ferulic acid (0.914), and negatively correlated to the anthocyanin $(<-0.900)$. The results indicated that phenolic acids (caffeic acid, salicylic acid) and flavonoids (quercetin) and resveratrol from blueberries played the main roles in the regulation of protein expressions.

\section{Correlation of gene expressions and ROS}

In this study the inhibition of the ROS production and inflammatory cytokines were first detected in LPS-activated macrophages. Different varieties of blueberry extracts had different inhibitory effects. Our results suggest that the ROS may exert to suppress NLRP3 inflammasome activation, in turn inhibiting IL-1 $\beta$ secretion, Caspase-1 cleavage and ASC pyroptosome formation.

Correlations among the expressions of ${ }_{M u s} N L R P 3,{ }_{M u s} I L-1 \beta$, Mus $A S C$, MusTNF- $\alpha$, Mus IL-6, MusiNOS and ROS by blueberry extracts were analyzed as shown in Table 3. ${ }_{\text {Mus }} A S C$ were highly positively correlated with the expressions of ${ }_{M u s} N L R P 3$ and ${ }_{M u s} I L-1 \beta$. ${ }_{\text {Mus }} I L-6$ was highly correlation with the expression of ${ }_{M u s} I L-1 \beta$, and ${ }_{M u s} A S C$. ${ }_{M u s} i N O S$ were highly correlated to the

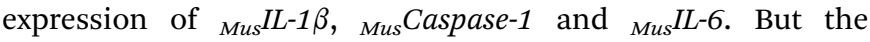
expression of the ROS was not strongly correlated to the expression of the mRNA and the highest correlation value was 0.304 with the ${ }_{M u s} T N F-\alpha$. 

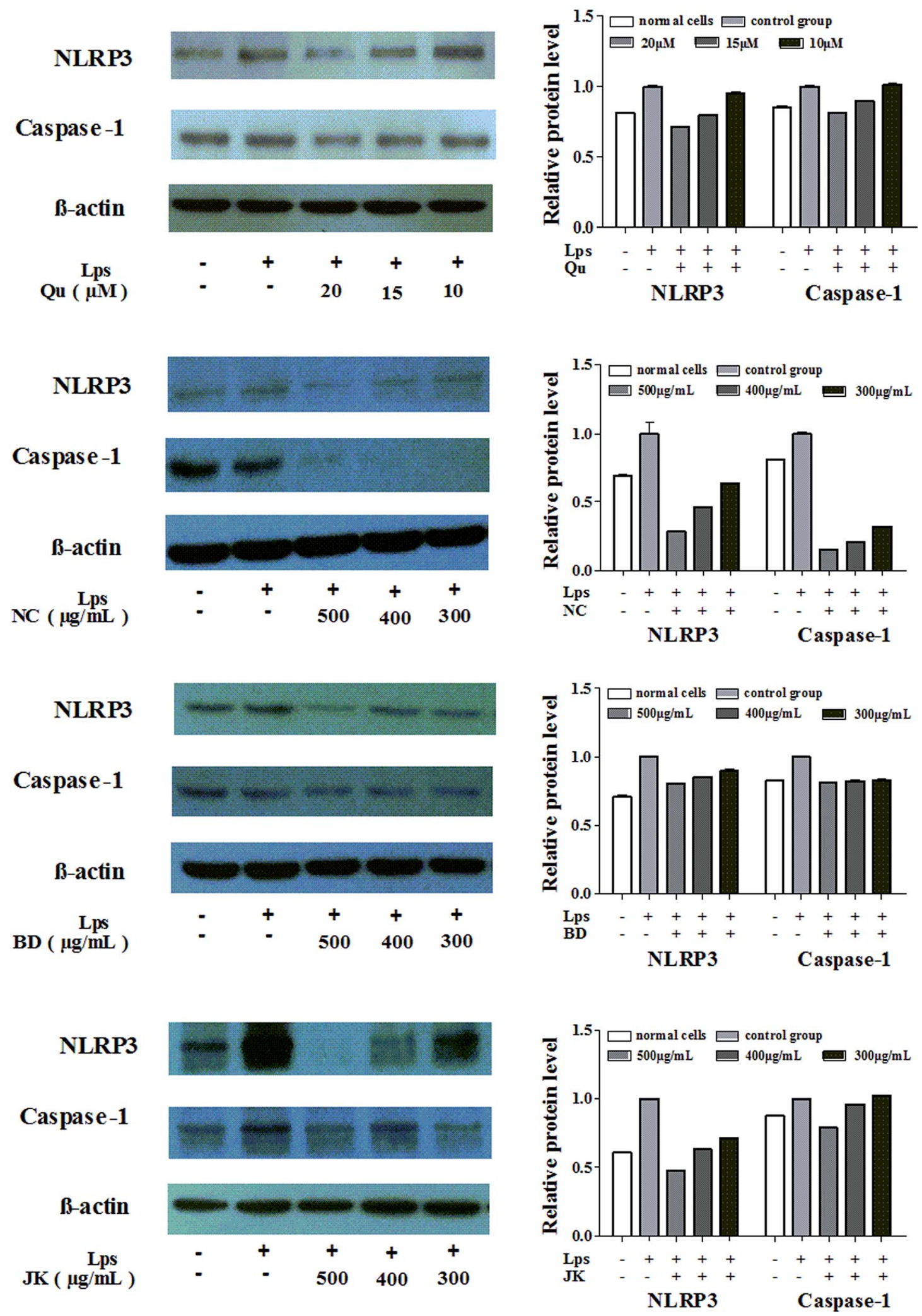

Fig. 3 The relative expression level of NLRP3 and Caspase-1 protein in mononuclear macrophage Raw264.7 cells (mean \pm SD, $n=3$ ). 
Table 3 Correlation of gene expression and ROS in blueberry extracts (Pearson test, $p<0.05$ )

\begin{tabular}{|c|c|c|c|c|c|c|c|c|}
\hline Correlation & ${ }_{M u s} N L R P 3$ & MusCaspase-1 & ${ }_{M u s} I L-1 \beta$ & ${ }_{M u s} A S C$ & Mus $T N F-\alpha$ & Mus $I L-6$ & MusiNOS & ROS \\
\hline Mus $N L R P 3$ & 1 & 0.726 & 0.746 & 0.841 & -0.173 & 0.553 & 0.456 & -0.716 \\
\hline MusCaspase-1 & & 1 & 0.844 & 0.838 & -0.124 & 0.791 & 0.891 & -0.174 \\
\hline${ }_{M u S} A S C$ & & & & 1 & -0.249 & 0.783 & 0.756 & -0.456 \\
\hline Mus TNF- $\alpha$ & & & & & 1 & 0.220 & 0.041 & 0.304 \\
\hline Mus IL-6 & & & & & & 1 & 0.860 & -0.016 \\
\hline
\end{tabular}

\section{Experimental}

\section{Chemicals and ingredients}

Trizol Regents, LPS, quercetin, and dichlorofluorescin diacetate (DCFH-DA) were purchased from Sigma Aldrich (St. Louis, MO, USA). Mus macrophage cell RAW264.7 (ATCC TIB-71) was purchased from the American Tissue Culture Collection (ATCC, Manassas, VA, USA). Phosphate buffer solution (PBS), cell culture medium (DMEM), fetal bovine serum (FBS) were purchased from GIBCO (Life Technologies, Grand Island, NY). iTaq Universal SYBR Green Super-mix, NLRP3 and Caspase-1 antibody were purchased from Boshide Company (Wuhan, China). The gene-specific primers were synthesized from Sangon Biotech Co. Ltd. (Shanghai, China).

\section{Blueberries samples and standards preparation}

Three varieties of fresh blueberries including: Blomidom (BD), North Country (NC) and JK-M7 (JK) were achieved from Jikang Company in Jilin province, China in 2015. Samples were prepared using the methods as reported previously. ${ }^{42}$ Quercetin (Qu) was used as standard for analysis.

\section{RAW264.7 macrophages cells cytotoxicity}

The cytotoxicity activity effects of blueberry were detected with the methods as previously described ${ }^{39}$ using RAW264.7 cells plated in 96 well plates with a concentration of $1 \times 10^{5}$ cells per well and incubated with blueberry extracts for $24 \mathrm{~h}$ at $37^{\circ} \mathrm{C}$. At the end of treatment, the medium that control and blueberrytreated group were discard and added methylene blue solution which was used to stain the cells in order to determine the survival rate of cells.

\section{ROS detection}

LPS and the blueberry extracts were used to induce the RAW264.7 cells. After incubated $12 \mathrm{~h}$, intracellular ROS was measured with the ROS-specific fluorescent probe DCFH-DA (20 mM; Molecular Probes) using the method described previously in our laboratory. ${ }^{28}$ RAW264.7 cells were loaded for $30 \mathrm{~min}$ with $20 \mathrm{mM}$ DCFH-DA, and washed twice with PBS, then read the fluorescent values using a multi-mode microplate reader (Filter Max F5, Molecular Devices, USA) with $485 \mathrm{~nm}$ excitation and $538 \mathrm{~nm}$ emission. The level of fluorescence was determined by Fluorescent Ascent FL plate-reader $\left(37^{\circ} \mathrm{C}\right) .{ }^{45}$

\section{Quantitative real-time RT-PCR analysis of gene expression}

Total RNA were isolated from the control and treated cells using Trizol Regents. The quantity of total RNA was determined by a spectrophotometer using the absorbance at $A_{260} / A_{280} \mathrm{~nm}$. cDNA was generated using the Prime Script TM RT reagent Kit (Takara, Japan). Quantitative real-time PCR were carried out using SYBR Green Super-mix for real-time PCR in triplicates and was performed using the Bio-Rad MiniOption ${ }^{\mathrm{TM}}$ Real Time PCR Detection System (Bio-Rad, Hercules, CA, USA). The primer sequences for RT-qPCR as Table 1 . The thermal cycle conditions were optimized as $10 \mathrm{~s}$ at $95{ }^{\circ} \mathrm{C}$ followed by 40 cycles of amplification $\left(5 \mathrm{~s}\right.$ at $95{ }^{\circ} \mathrm{C}$ and $30 \mathrm{~s}$ at $\left.60{ }^{\circ} \mathrm{C}\right)$. The relative expression of each gene was calculated using the comparative threshold cycle method normalized to ${ }_{M u s} G A P D H$ (reference gene). Analysis of relative gene expression data using real-time quantitative PCR and the $2^{-\Delta \Delta C_{\mathrm{t}}}$ method. The results were reported as mean $\pm \mathrm{SD}(n=3)$.

\section{Western-blot for NLRP3 inflammasome containing NLRP3, Caspase-1}

Protein extraction for Western blot was conducted using the method reported previously. ${ }^{\mathbf{4 6}}$ Cells were rinsed twice in ice-cold PBS, and then added cell lysis buffer containing $1 \mathrm{mM}$ PMSF pyrolysis $5 \mathrm{~min}$, harvested and kept on ice for $30 \mathrm{~min}$ in with constant agitation. Insoluble cell debris was discarded following centrifugation for $15 \mathrm{~min}$ at $12000 \mathrm{rpm}$ at $4{ }^{\circ} \mathrm{C}$. The protein samples were separated by sodium dodecyl sulfate polyacrylamide gel electrophoresis (SDS-PAGE) on 10\% gels and subsequently transferred to polyvinylidene (PVDF) membranes (Millipore). ${ }^{46}$ Immunoblotting was performed for NLRP3, Caspase-1 with $\beta$-actin expression as an internal control.

\section{Statistical analysis}

The evaluation of statistical significance of observed differences was performed by one-way analysis of variance (one-way ANOVA), using SPSS software 21.0 (SPSS Inc., Chicago, IL, USA), statistical significance was set at $p<0.05$. The graphs were used with the Graph Pad INSTAT software (GraphPad software, San Diego, CA, USA).

\section{Conclusions}

Blueberry had been reported to have significant antioxidant activity using the method of the CAA, PSC, and ORAC. In this 
paper using mononuclear macrophage (RAW264.7) to detect the effects of the blueberry extracts on anti-inflammation activities. The mononuclear macrophage was treated with the blueberry extracts and quercetin after LPS stimulation, and the expressions of inflammatory factors and cytokines were detected. Blueberry extracts significantly inhibited genes expression of Mus $I L-1 \beta,{ }_{M u s} N L R P 3,{ }_{\text {Mus }}$ Caspase-1, ${ }_{\text {Mus }} A S C,{ }_{M u s} T N F-\alpha,{ }_{M u s} I L-6$, and ${ }_{\text {Mus }}$ iNOS. ${ }_{\text {Mus }}$ NLRP3 and ${ }_{\text {Mus }}$ Caspase-1 were also detected from gene and protein levels and the blueberry treated groups were significantly lower than the LPS treated group. Meanwhile, quercetin significantly inhibited LPS-induced ROS in RAW264.7 cells. Furthermore, quercetin and blueberry extracts inhibited the protein expression of NLRP3 and Caspase-1. In conclusion, we used LPS stimulated RAW264.7 cells to produce ROS in mitochondria, and then induced mitochondrial dysfunction to further stimulate the NLRP3 inflammasome including the

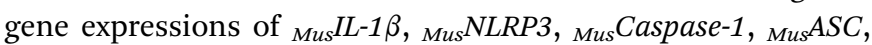
Mus TNF- $\alpha$, Mus IL-6, and ${ }_{\text {Mus }} i N O S$ and protein expressions of NLRP3 and Caspase-1. Our results suggest that bioactive compounds of blueberries may provide health benefits with anti-inflammatory potentials.

\section{Acknowledgements}

Authors are greatly thankful to National Natural Science Foundation of China (31501765), Science and Technology Planning Project of Guangdong-China (2016B020233004), Natural Science Foundation of Guangdong-China (2016A030312001) and the Leading Talents Program in Guangdong Province for financial support, valuable suggestions and guidelines.

\section{References}

1 X. Liu, L. Fang, T. B. Guo, H. Mei and J. Z. Zhang, Trends Immunol., 2013, 34, 120-128.

2 P. Xi and R. H. Liu, Mol. Nutr. Food Res., 2016, 60, 1819-1836.

3 X. Jiang, T. Li and R. H. Liu, J. Agric. Food Chem., 2016, 64, 1806-1816.

4 L. Franchi, T. Eigenbrod, R. Muñoz-Planillo and G. Nuñez, Nat. Immunol., 2009, 10, 241-247.

5 F. Martinon, A. Mayor and J. Tschopp, Annu. Rev. Immunol., 2009, 27, 229-265.

6 S. Mariathasan, K. Newton, D. M. Monack, D. Vucic, D. M. French, W. P. Lee, M. Roose-Girma, S. Erickson and V. M. Dixit, Nature, 2004, 430, 213-218.

7 I. C. Allen, M. A. Scull, C. B. Moore, E. K. Holl, E. McElvaniaTeKippe, D. J. Taxman, E. H. Guthrie, R. J. Pickles and J. P.-Y. Ting, Immunity, 2009, 30, 556-565.

8 P. G. Thomas, P. Dash, J. R. Aldridge, A. H. Ellebedy, C. Reynolds, A. J. Funk, W. J. Martin, M. Lamkanfi, R. J. Webby and K. L. Boyd, Immunity, 2009, 30, 566-575.

9 O. Gross, H. Poeck, M. Bscheider, C. Dostert, N. Hannesschläger, S. Endres, G. Hartmann, A. Tardivel, E. Schweighoffer and V. Tybulewicz, Nature, 2009, 459, 433-436.

10 S. L. Cassel, S. C. Eisenbarth, S. S. Iyer, J. J. Sadler, O. R. Colegio, L. A. Tephly, A. B. Carter, P. B. Rothman,
R. A. Flavell and F. S. Sutterwala, Proc. Natl. Acad. Sci. U. S. A., 2008, 105, 9035-9040.

11 F. Martinon, V. Pétrilli, A. Mayor, A. Tardivel and J. Tschopp, Nature, 2006, 440, 237-241.

12 C. Dostert, V. Pétrilli, R. Van Bruggen, C. Steele, B. T. Mossman and J. Tschopp, Science, 2008, 320, 674677.

13 V. Hornung, F. Bauernfeind, A. Halle, E. O. Samstad, H. Kono, K. L. Rock, K. A. Fitzgerald and E. Latz, Nat. Immunol., 2008, 9, 847-856.

14 D. A. Muruve, V. Pétrilli, A. K. Zaiss, L. R. White, S. A. Clark, P. J. Ross, R. J. Parks and J. Tschopp, Nature, 2008, 452, 103107.

15 I. Aksentijevich, C. D Putnam, E. F. Remmers, J. L. Mueller, J. Le, R. D. Kolodner, Z. Moak, M. Chuang, F. Austin and R. Goldbach-Mansky, Arthritis Rheum., 2007, 56, 1273-1285. 16 K. Shinkai, T. McCalmont and K. Leslie, Clin. Exp. Dermatol., 2008, 33, 1-9.

17 K. Schroder and J. Tschopp, Cell, 2010, 140, 821-832.

18 F. Martinon, K. Burns and J. Tschopp, Mol. Cell, 2002, 10, 417-426.

19 S. D. Brydges, J. L. Mueller, M. D. McGeough, C. A. Pena, A. Misaghi, C. Gandhi, C. D. Putnam, D. L. Boyle, G. S. Firestein and A. A. Horner, Immunity, 2009, 30, 875-887.

20 G. Meng, F. Zhang, I. Fuss, A. Kitani and W. Strober, Immunity, 2009, 30, 860-874.

21 S. Mariathasan, D. S. Weiss, K. Newton, J. McBride, K. O'Rourke, M. Roose-Girma, W. P. Lee, Y. Weinrauch, D. M. Monack and V. M. Dixit, Nature, 2006, 440, 228232.

22 C. Xie, J. Kang, M. E. Ferguson, S. Nagarajan, T. M. Badger and X. Wu, Mol. Nutr. Food Res., 2011, 55, 1587-1591.

23 A. Cheng, H. Yan, C. Han, W. Wang, Y. Tian and X. Chen, Int. J. Biol. Macromol., 2014, 69, 382-387.

24 T. Misawa, T. Saitoh, T. Kozaki, S. Park, M. Takahama and S. Akira, Int. Immunol., 2015, 27, 425-434.

25 J. Kang, K. M. Thakali, G. S. Jensen and X. Wu, Plant Foods Hum. Nutr., 2015, 70, 56-62.

26 K. L. Wolfe and R. H. Liu, J. Agric. Food Chem., 2008, 56, 8404-8411.

27 X. Sun, N. Liu, Z. Wu, Y. Feng and X. Meng, Molecules, 2015, 20, 3841-3853.

28 K. L. Wolfe, X. Kang, X. He, M. Dong, Q. Zhang and R. H. Liu, J. Agric. Food Chem., 2008, 56, 8418-8426.

29 Z. Diaconeasa, L. Leopold, D. Rugină, H. Ayvaz and C. Socaciu, Int. J. Mol. Sci., 2015, 16, 2352-2365.

30 A. Ben Lagha, S. Dudonne, Y. Desjardins and D. Grenier, J. Agric. Food Chem., 2015, 63, 6999-7008.

31 M. Okamoto, W. Liu, Y. Luo, A. Tanaka, X. Cai, D. A. Norris, C. A. Dinarello and M. Fujita, J. Biol. Chem., 2010, 285, 64776488.

32 P. Limtrakul, S. Yodkeeree, P. Pitchakarn and W. Punfa, Asian Pac. J. Cancer Prev., 2015, 16, 4277-4283.

33 W. Y. Huang, Y. M. Liu, J. Wang, X. N. Wang and C. Y. Li, Molecules, 2014, 19, 12827-12841.

34 W.-Y. Huang, J. Wang, Y.-M. Liu, Q.-S. Zheng and C.-Y. Li, Eur. J. Pharmacol., 2014, 723, 67-72. 
35 G. Flores, K. Dastmalchi, A. J. Dabo, K. Whalen, P. PedrazaPenalosa, R. F. Foronjy, J. M. D'Armiento and E. J. Kennelly, Food Chem., 2012, 131, 119-125.

36 V. Taverniti, D. Fracassetti, C. Del Bo, C. Lanti, M. Minuzzo, D. Klimis-Zacas, P. Riso and S. Guglielmetti, J. Agric. Food Chem., 2014, 62, 8346-8351.

37 M. H. Johnson, E. G. de Mejia, J. Fan, M. A. Lila and G. G. Yousef, Mol. Nutr. Food Res., 2013, 57, 1182-1197.

38 J. Boyer, D. Brown and R. H. Liu, J. Agric. Food Chem., 2004, 52, 7172-7179.

39 D. L. Felice, J. Sun and R. H. Liu, J. Funct. Foods, 2009, 1, 109118.
40 D. F. Garcia-Diaz, M. H. Johnson and E. G. de Mejia, J. Med. Food, 2015, 18, 489-496.

41 R. H. Liu, J. Food Sci., 2013, 78, A18-A25.

42 H. Wang, X. Guo, X. Hu, T. Li, X. Fu and R. H. Liu, Food Chem., 2017, 217, 773-781.

43 W. W. Chao, Y. C. Chung, I. P. Shih, H. Y. Wang, S. T. Chou and C. K. Hsu, J. Med. Food, 2015, 18, 724-730.

44 T. Tsuda, Mol. Nutr. Food Res., 2012, 56, 159-170.

45 K. L. Wolfe and R. H. Liu, J. Agric. Food Chem., 2007, 55, 8896-8907.

46 R. H. Liu, J. Jacob and B. Tennant, BioTechniques, 1997, 22, 594-595. 